\title{
The Impact of International Quality Assurance on Mexican Higher Education
}

\author{
Dr. Enrique A. Morales González ${ }^{1}$, Dr. José Humberto Loría Arcila², \\ Mtra. Margarita Díaz Olaldez ${ }^{1}$, Ing. Miriam del C. Flores Castillo ${ }^{1}$ \\ ${ }^{1}$ Universidad Veracruzana, México, emorales@uv.mx,mardiaz@uv.mx, mirflores@uv.mx \\ ${ }^{2}$ Universidad Autónoma de Yucatán, México, jose.loria@uady.mx
}

\begin{abstract}
This document describes the international trends in educational field and its impact on the quality of Higher Education for engineering in Mexico. Due to the expansion of student enrollment in higher education, a wide education availability, new technologies and emerging global networks, higher education has been involved in a constant change of methods to demonstrate quality on national and international level.

In this paper, the term "quality" is defined as a comparison reference between several homologous elements or under a certain reference standard. Besides, the concept of Quality Assurance is presented beneath two scopes: responsibility and improvement; The first one is focused on the accountability that Higher Education Institutions must do to the State and Society; And the second one is focused in the internal control and continuous improvement of the effectiveness of education. In other words; there are two principal perspectives: an external approach and an internal approach. This last view is taken by most countries in America and Europe, promoting the creation of bodies responsible for accrediting engineering programs in international level.

In Mexico, there are more than 70 higher education engineering programs accredited by at least an internationally recognized accreditation association. Considering the principal accreditation associations on Canada, United States, Mexico, Europe and Latin America, a comparison is made of the main criteria and standards with which they evaluate each of the different organisms to engineering programs in order to identify similarities.
\end{abstract}

Keywords-Quality, Accreditation, Mexico, Higher Education, International

Digital Object Identifier (DOI):

http://dx.doi.org/10.18687/LACCEI2016.1.1.400

ISBN: 978-0-9822896-9-3

ISSN: 2414-6390

$14^{\text {th }}$ LACCEI International Multi-Conference for Engineering, Education, and Technology: "Engineering Innovations for Global Sustainability", 20-22 July 2016, San José, Costa Rica. 


\title{
The Impact of International Quality Assurance on Mexican Higher Education
}

\author{
Dr. Enrique A. Morales González ${ }^{1}$, Dr. José Humberto Loría Arcila ${ }^{2}$, \\ Mtra. Margarita Díaz Olaldez ${ }^{1}$, Ing. Miriam del C. Flores Castillo ${ }^{1}$ \\ ${ }^{1}$ Universidad Veracruzana, México, emorales@uv.mx, mardiaz@uv.mx, mirflores@uv.mx \\ ${ }^{2}$ Universidad Autónoma de Yucatán, México, jose.loria@uady.mx
}

\begin{abstract}
This document describes the international trends in educational field and its impact on the quality of Higher Education for engineering in Mexico. Due to the expansion of student enrollment in higher education, a wide education availability, new technologies and emerging global networks, higher education has been involved in a constant change of methods to demonstrate quality on national and international level.

In this paper, the term "quality" is defined as a comparison reference between several homologous elements or under a certain reference standard. Besides, the concept of Quality Assurance is presented beneath two scopes: responsibility and improvement; The first one is focused on the accountability that Higher Education Institutions must do to the State and Society; And the second one is focused in the internal control and continuous improvement of the effectiveness of education. In other words; there are two principal perspectives: an external approach and an internal approach. This last view is taken by most countries in America and Europe, promoting the creation of bodies responsible for accrediting engineering programs in international level.
\end{abstract}

In Mexico, there are more than 70 higher education engineering programs accredited by at least an internationally recognized accreditation association. Considering the principal accreditation associations on Canada, United States, Mexico, Europe and Latin America, a comparison is made of the main criteria and standards with which they evaluate each of the different organisms to engineering programs in order to identify similarities.

Keywords-Quality, Accreditation, Mexico, Higher Education, International
Resumen- El presente documento describe las tendencias internacionales en el ámbito educativo y su impacto en la calidad de la Educación Superior para la ingeniería en México. Tras la expansión de la matrícula de estudiantes en educación superior, la diversidad en la oferta educativa, las nuevas tecnologías y las redes globales emergentes, la educación superior se ha visto envuelta en un constante cambio en los métodos de demostrar la calidad a nivel nacional e internacional.

Se define el término "calidad" como una referencia de comparación entre varios elementos homologables o bajo cierto patrón de referencia. Por otro lado, el concepto de Aseguramiento de Calidad se aborda bajo dos expectativas: responsabilidad y mejora; el primero, enfocado a la rendición de cuentas que las Instituciones de Educación Superior (IES) deben hacer al Estado o la sociedad; y el segundo, al control interno y al mejoramiento continuo de la efectividad de la educación, es decir; existen dos perspectivas principales; un enfoque externo y un enfoque interno. Esta última visión es la que es tomada por la mayoría de los países de América y Europa, promoviendo la creación de organismos encargados de acreditar a los programas de ingeniería a nivel internacional.

En México, existen más de 70 programas de ingeniería de educación superior acreditadas por, al menos, un organismo de acreditación con reconocimiento internacional. Tomando en cuenta los principales organismos de acreditación de los países de Canadá, Estados Unidos, México, Europa y América Latina, se hace una comparativa de los principales criterios y estándares con los que evalúan cada uno de los distintos organismos a los programas de ingeniería con el fin de identificar las similitudes.

Palabras Claves: Calidad, Acreditación, México, Educación Superior, Internacionalización

Digital Object Identifier (DOI):

http://dx.doi.org/10.18687/LACCEI2016.1.1.400

ISBN: 978-0-9822896-9-3

ISSN: $2414-6390$

$14^{\text {th }}$ LACCEI International Multi-Conference for Engineering, Education, and Technology: "Engineering Innovations for Global Sustainability", 20-22 July 2016, San José, Costa Rica. 


\title{
Impacto de la Calidad Internacional en la Educación Superior de México
}

\author{
Dr. Enrique A. Morales González¹, José Humberto Loría Arcila², \\ Mtra. Margarita Díaz Olaldez ${ }^{1}$ Ing. Miriam del C. Flores Castillo ${ }^{1}$ \\ 1'Universidad Veracruzana, México, emorales@uv.mx, mardiaz@uv.mx, mirflores@uv.mx \\ ${ }^{2}$ Universidad Autónoma de Yucatán, México, jose.loria@uady.mx
}

\section{ANTECEDENTES}

La educación se encuentra en constante cambio afectada por el ambiente social, económico y cultural en el que se desenvuelve. En sus comienzos, la educación superior estaba centrada en la enseñanza de teorías y conceptos. Actualmente, además del aprendizaje de la teoría, está especialmente enfocada en la formación de egresados con un alto grado de experiencia laboral y prácticas; así como la formación en habilidades y aptitudes destinadas a un mejor desempeño laboral y social.

Según el Centro Interuniversitario de Desarrollo (CINDA) [1], la situación de la educación superior está regida por corrientes o tendencias de acuerdo a su entorno económico, social y cultural, las cuales son: a) expansión de la educación superior, b) diversificación de la oferta, c) población estudiantil más heterogénea, d) colaboración, movilidad y redes globales y e) atención creciente a la responsabilidad pública y el desempeño.

Considerando dichas corrientes, así como otras fuentes de información se puede describir lo siguiente:

\section{A. Expansión de la educación superior}

El acceso a la educación superior se expandió aceleradamente en la región de Latinoamérica y el Caribe durante la década del 2000, llegando a un promedio de crecimiento de aproximadamente $40 \%$, lo que permitió que la región se situara en el promedio de la tendencia internacional. [2]

En México, en los últimos 20 años, se ha duplicado el total de jóvenes matriculados en escuelas de educación superior, pasando de poco más de 1 millón y medio en 1996 a más de 3 millones y medio en el año 2014. [3]

\section{B. Diversificación de la oferta}

La diversificación tiene diferentes aspectos: la creación de nuevos tipos de instituciones, la multiplicación de ofertas educacionales, la expansión de la oferta privada y la introducción de nuevas formas de prestación de servicio. [1]

Debido a esto, en toda América Latina se ha incrementado la apertura de instituciones; tanto públicas como privadas, que ofrecen distintos métodos de enseñanza, o modelos educativos, que proponen aspectos llamativos como: horarios flexibles, movilidad nacional e internacional, talleres y laboratorios especializados, entre otros; con el fin de lograr una mayor cobertura de estudiantes.

$14^{\text {th }}$ LACCEI International Multi-Conference for Engineering, Education, and Technology: "Engineering Innovations for Global Sustainability”, 20-22 July 2016, San José, Costa Rica.

\section{Población estudiantil más heterogénea}

La expansión de la población estudiantil no sólo significa mayor número, sino mayor diversidad: edad, género, aptitud, estrato social, capital cultural y expectativas; estos son factores de diferenciación, que hacen que para un número considerable de instituciones de educación superior (IES), acostumbradas a tratar con alumnos tradicionales, es decir, principalmente varones jóvenes, altamente calificados y que aspiran a una carrera académica o profesional, resulte difícil ajustarse a estas nuevas necesidades y demandas. [1]

\section{Nuevas tecnologías}

Las nuevas tecnologías se desplazan a escala global, y los Estados nacionales parecen tener limitaciones técnicas y estructurales para regular los flujos existentes y también para contraponer dinámicas o intenciones en esa velocidad y escala. Sin embargo, en los últimos años puede observarse que hay una recuperación de la iniciativa por parte de los Estados que han desarrollado diferentes programas orientados a equipar, capacitar e incorporar en el territorio escolar las nuevas tecnologías.

Las experiencias son muy diversas, como también lo han sido sus objetivos y grados de desarrollo, pero ya podemos señalar una serie de iniciativas muy valorables para América Latina, como el Plan Ceibal en Uruguay, Enlaces en Chile, Proyecto Huascarán en Perú, Programa Computadoras para Educar en Colombia, Programa integral Conéctate en El Salvador, Escuelas del Futuro en Guatemala o el Plan de Inclusión Digital Educativa y Conectar Igualdad en la Argentina, entre otros. Todos ellos dan muestras de la existencia de políticas que buscan acompañar estas transformaciones, sumarlas al mundo de la escuela y orientarlas en una dirección determinada. [4]

\section{E. Colaboración, movilidad y redes globales}

El aumento de la internacionalización de la educación superior tiene varios aspectos que es preciso mencionar.

- Movilidad de académicos, estudiantes y profesionales, la que a su vez se relaciona con plan de estudios, al menos en áreas tales como ingeniería, comercio y gestión, informática y biotecnología.

- Movilidad de la educación, es decir que la institución tenga la capacidad de brindar sus programas presenciales a través de mecanismos de aprendizaje electrónico, o de revalidar sus programas con otros programas de otras instituciones nacionales o internacionales.
(- 
- La colaboración internacional y las redes entre instituciones en diferentes áreas de enseñanza e investigación. [1]

\section{F. Atención creciente a la responsabilidad pública y el desempeño}

La expansión de los sistemas de educación superior, su mayor diversificación, la necesidad de legitimar el uso de fondos públicos y el aumento de las presiones del mercado son factores que exigen una mayor inspección sobre la educación superior porque su calidad, eficacia y eficiencia ya no se dan por sentado, sino que se deben demostrar y verificar. [1]

Sin duda, la preocupación por la calidad de la educación superior y por su aseguramiento por parte de autoridades estatales, líderes institucionales o las propias instituciones no son nuevas prácticas, pero antes estaban restringidas al interior del sistema de educación superior en sí. Lo que es nuevo es la importancia social que se otorga a su calidad y por ende, la necesidad de las IES de encontrar nuevos asociados y desarrollar vínculos con el entorno social y productivo, a fin de poder identificar y encontrar respuestas adecuadas a las necesidades sociales. [1] Así como poner énfasis a temas actuales considerados como relevantes para la humanidad: sustentabilidad, ética, emprendimiento, responsabilidad social, entre otros.

\section{DESAFÍOS Y OPORTUNIDADES PARA LA EDUCACIÓN SUPERIOR EN AMÉRICA LATINA}

El primer desafío de los sistemas de educación de nivel superior es asegurar la equidad de su expansión, transformándose en impulsores de promoción y movilidad social. Por lo que los países deberán introducir criterios de equidad en el acceso que permitan al menos contextualizar el mérito, tomando en cuenta la condición social de origen de los jóvenes.

El segundo desafío es responder a las exigencias de globalización, lo que representa, para los países en vías de desarrollo, generar su propia producción científica básica, promover la investigación aplicada y aumentar la inscripción de patentes.

El tercer desafío es la relación con la sociedad. Los sistemas de educación superior, para coordinarse internamente y vincularse con otras esferas sociales, necesitan una mayor racionalización y mejores mecanismos de rendición de cuentas hacia la sociedad. Esto no implica anular la autonomía de las instituciones, sino crear instrumentos de política pública que permitan a la sociedad, a través de sus instituciones, monitorearlas y orientarlas hacia fines públicos. Sin duda, los sistemas de acreditación y evaluación que se han venido construyendo en las últimas décadas pueden contribuir significativamente a este propósito. [2]

A nivel internacional se están estableciendo mecanismos de acreditación en carreras en las que el título universitario sea requisito o condición para el ejercicio de la profesión y se han creado redes en áreas específicas, tales como Ingeniería, Medicina o Agronomía. Existe una tendencia a que estas carreras consideradas de "riesgo social" se regulen con mayor énfasis. Con el auge de los procesos de evaluación, reconocimiento y mejoramiento, las carreras de Ingeniería se ven implicadas en iniciativas regionales para llegar a un consenso con respecto a los requerimientos de calidad que deberían cumplir estas carreras y los mecanismos de evaluación de éstos. [5]

Como consecuencia de estos cambios, la mayoría de los países han instalado, o se encuentra instalando, mecanismos de aseguramiento que den garantía pública acerca de la calidad de las instituciones de educación superior y de las carreras y programas que ofrecen. Estos mecanismos de aseguramiento que son acreditados de forma nacional o internacional demuestran, de manera objetiva, la capacidad que tiene una institución en alcanzar los objetivos de su organización. [1]

\section{SISTEMA INTERNO DE ASEGURAMIENTO DE CALIDAD}

Para comprender el término "Sistema Interno de Aseguramiento de Calidad" es necesario definir, en primera instancia, los siguientes conceptos: a) Calidad desde el ámbito de "Educación Superior"; b) qué es un "Sistema de Aseguramiento de Calidad" y sus dos enfoques principales: Responsabilidad y Mejora; y c) el concepto de "Sistema Interno de Aseguramiento de Calidad" en sí.

\section{A. Calidad}

La calidad es un término relativo debido a varias razones; es relativo para quien usa el término y es relativo dependiendo de las circunstancias en las cuales se hace uso. Algunos autores distinguen hasta siete concepciones diferentes de calidad definidas por el enfoque al que van encaminadas. Estas son: como excelencia, como perfección, como cumplimiento del propósito institucional, como transformación del estudiante, como cota mínima, como justiprecio y como mejoramiento continuo. [6]

Debido a la complejidad de la palabra, se toma como referencia la concepción hecha por el Centro Interuniversitario de Desarrollo (CINDA), que postula "el concepto de calidad en la educación superior no existe como tal, sino como un término de referencia de carácter comparativo en el cual algo puede ser mejor o peor que otro, dentro de un conjunto de elementos homologables, o en comparación con cierto patrón de referencia -real o utópico- previamente determinado". [1]

\section{B. Aseguramiento de la Calidad: Responsabilidad y Mejora}

En términos generales los procesos de aseguramiento de la calidad pueden abarcar procesos internos de mejoramiento continuo de calidad en las propias instituciones, o bien, concentrarse en mecanismos de verificación de cumplimiento de estándares mínimos de calidad previamente validados. En este sentido, mejoramiento de calidad se asocia a alcanzar un mínimo de calidad en un momento del tiempo y también a alcanzar un mejoramiento continuo y permanente de calidad. [7]

En este marco, destacan dos perspectivas o enfoques desde los cuales se intenta asegurar la calidad:

1. Aseguramiento de la calidad enfocado hacia la mayor transparencia y rendición de cuentas (Accountability): Perspectiva asociada al control de estructuras administrativas y verificación externa del cumplimiento de estándares mínimos. Se relaciona con la transparencia de la información por la

14 ${ }^{\text {th }}$ LACCEI International Multi-Conference for Engineering, Education, and Technology: "Engineering Innovations for Global Sustainability”, 20-22 July 2016, San José, Costa Rica. 
necesidad de garantizar el correcto uso de recursos públicos y para salvaguardar el interés social asegurando estándares de la Educación Superior y generar niveles de garantía o confianza proporcionando información independiente y verificable de programas e IES.

2. Aseguramiento de calidad para el mejoramiento permanente (Improvement): Enfoque asociado al control interno y a la autorregulación de las IES para el mejoramiento continuo de la efectividad de la educación superior, permitiendo al mundo académico revisar sus aproximaciones, métodos, y analizar sus fortalezas y debilidades. Las instituciones o programas se plantean metas de superación continua, para lo cual se requiere haber superado los estándares mínimos e incorporarse a procesos voluntarios de mejoramiento permanente con ayuda de agentes externos.

La combinación de ambas perspectivas es común en varios países, como una tendencia a ir equilibrando entre formas de control externas y la responsabilidad de las IES por su propia calidad con mecanismos internos de monitoreo y evaluación que permitan el mejoramiento continuo. [7]

La "Mejora Continua" se define como un ciclo ininterrumpido, a través del cual se identifica un área de mejora, se establece cómo realizar un plan de mejora, se implementa, se verifican los resultados y se toman acciones de acuerdo con ellos, ya sea para corregir desviaciones o para proponer un objetivo más alto. Este ciclo permite la renovación, el desarrollo, el progreso y la posibilidad de responder a las necesidades cambiantes del entorno, para dar un mejor servicio o producto a los clientes o usuarios. Para llevar a cabo un plan de mejora continua es necesario que cada uno de los individuos involucrados en el proceso participe permanentemente, lo que implica una campaña de concientización para cada uno de los empleados y grupos de interés asociados a la organización. [8]

\section{Sistema de Aseguramiento Interno de la Calidad}

El Sistema de Garantía de la Calidad está integrado por dos subsistemas: El sistema interno de aseguramiento de la calidad y El sistema externo de aseguramiento de la calidad. El sistema interno de aseguramiento de la calidad lo desarrollan las IES públicas y particulares y los centros públicos de investigación y tiene como finalidad informar con transparencia a los interesados sobre la calidad de sus programas [9].

El Consejo Nacional de Ciencia y Tecnología (CONACYT) [10] señala que el Sistema Interno de Aseguramiento de Calidad se concibe como "un instrumento de autorregulación, innovación y desarrollo institucional, donde la comunidad y los directivos se hayan identificados con la innovación y el plan de mejora, y los estudiantes se han sumado al esfuerzo, asimismo se han incorporado a los interlocutores, internos y externos. Se ha desarrollado una buena comunicación mediante una discusión, franca, abierta y respetuosa, las acciones se apoyan en el uso de los mejores conocimientos disponibles, y se ha elaborado un marco de referencia dinámico para la mejora continua y la innovación, y se cuenta con indicadores, y procesos de autoevaluación y realimentación que se aplican de manera sistemática, así como de comparación con otras instituciones nacionales e $14^{\text {th }}$ LACCEI International Multi-Conference for Engineering, Education, and Technology: "Engineering Innovations for Global Sustainability”, 20-22 July 2016, San José, Costa Rica. internacionales, se han identificado las resistencias al cambio y se tienen estrategias para superarlas".

Contar con un Sistema Interno de Aseguramiento de la Calidad es una garantía para la institución de que los objetivos se cumplen satisfactoriamente, no sólo para sus fines institucionales sino también para la sociedad.

Un Sistema Interno de Aseguramiento de la Calidad basado en políticas, procedimientos, recursos y mecanismos ofrece un marco de orientación en las acciones de la institución en sus distintos procesos de forma sistemática lo que ayuda a consolidar buenas prácticas de calidad y volverlas tareas cotidianas de la institución.

En una institución de Educación Superior, el Sistema Interno de Aseguramiento de Calidad debe incluir a los estudiantes y profesores como principales protagonistas; los programas académicos como la ruta escogida para lograr los objetivos de la formación y la Gestión Académica, como los responsables de encontrar procesos que optimicen los procesos de toma de decisiones y de mejoramiento continuo. [11]

\section{ORGANISMOS DE ACREDITACIÓN PARA LA INGENIERÍA EN OTROS PAÍSES}

Durante las últimas décadas, en muchos países se han formado distintas agencias y organismos que se encargan del aseguramiento de la calidad en sus correspondientes países.

En Chile, en 1990, se creó el Consejo Superior de Educación (CSE) destinado a acreditar las instituciones privadas no autónomas (nuevas universidades privadas); en Colombia, en 1992, se originó el Consejo Nacional de Acreditación (CNA); en Brasil, en 1993, se puso en marcha el Programa de Evaluación Institucional de las Universidades Brasileñas (PAIUB) por iniciativa de las propias universidades con acuerdo con el Ministerio de Educación; en El Salvador se creó la Comisión Nacional de Acreditación (CDA); en Argentina, en 1995/96, se constituyó la Comisión Nacional de Evaluación y Acreditación Universitaria (CONEAU) y en Costa Rica, en 1999, se creó el Sistema Nacional de Acreditación de la Educación Superior (SINAES). Paralelamente, se establecieron entidades de evaluación y acreditación en otros países, tales como Uruguay, Bolivia y Panamá. [6]

En la región de Norteamérica se puede mencionar a Estados Unidos, donde, desde 1932, se estableció el Accreditation Board for Engineering and Technology (ABET) para la acreditación de programas de Ingeniería. En Canadá, se estableció en 1965 el Canadian Engineering Accreditation Board (CEAB), un órgano similar a ABET en metodología, organización y procedimientos. Tanto el ABET como el CEAB participan en el Acuerdo de Washington (Washington Accord) que se estableció en 1989, con la participación de Australia, Canadá, EEUU, Hong Kong, Irlanda, Nueva Zelanda, Sudáfrica, Reino Unido, Japón y Singapur [5]. Actualmente son 18 los países signatarios: Australia, Canadá, China, China Tapei, Corea, Estados Unidos, Hong Kong, India, Irlanda, Japón, Malasia, Nueva Zelanda, Reino Unido, Rusia, Singapur, Sri Lanka, Sudáfrica y Turquía. Adicionalmente, hay 6 países con el estatus de miembro provisional: Bangladesh, Costa Rica, Filpinas, México, Paquistán y Perú. [12] 
En Europa, desde 2002 se fundó La Agencia Nacional de Evaluación de la Calidad y Acreditación de España (ANECA), el cual es un organismo autónomo perteneciente a la Unión Europea que tiene como objetivo aportar garantía externa de calidad al sistema universitario y contribuir a su mejora constante. Europa también cuenta con otros organismos internacionales como: European Consortium for Accreditation (ECA), Red Iberoamericana para la Acreditación de la Calidad de la Educación Superior (RIACES), International Network for Quality Assurance Agencies in Higher Education (INQAAHE) y European Association for Quality Assurance in Higher Education (ENQA). Por otro lado, en 2006 La Red Europea para la Acreditación de la Enseñanza de la Ingeniería (ENAEE por sus siglas en inglés, European Network for Accreditation of Engineering Education) fue fundada después de la conclusión del proyecto EUR-ACE formado por 14 asociaciones europeas relacionadas con la educación de la ingeniería. [9]

También, existen acuerdos internacionales entre distintos países. Una de los más importantes en América es la Alianza Internacional de Ingeniería "IEA" por sus siglas en inglés (International Engineering Alliance), la cual se formó en 2007 cuando los acuerdos educativos y los acuerdos de movilidad vieron la necesidad de crear una Secretaría común y otras sinergias como la racionalización de sus reglas y directrices. En dicha alianza existen 7 acuerdos internacionales, de los cuales destacan: El Washington Accord, el Dublin Accord y el Sydney Accord [12].

El Washington Accord que se define a sí mismo como "un acuerdo multilateral entre agencias responsables de acreditación de los programas profesionales de ingeniería dentro de su jurisdicción, quienes han decidido trabajar colectivamente para asistir la movilidad de ingenieros profesionales que posean las calificaciones apropiadas". Fue firmado originalmente en el año 1989 por agencias acreditadoras en ingeniería de seis países para reconocer la equivalencia substancial de los programas de Ingeniería acreditados entre dichas agencias.

El Dublín Accord es un acuerdo para el reconocimiento internacional de las cualificaciones de nivel técnico de la ingeniería. En mayo de 2002, las organizaciones nacionales de ingeniería del Reino Unido, República de Irlanda, Sudáfrica y Canadá han firmado un acuerdo de reconocimiento mutuo de las cualificaciones que se basa la concesión de títulos técnicos de ingeniería en los cuatro países. Actualmente hay ocho firmantes del Acuerdo de Dublín; Australia, Canadá, Irlanda, Corea, Nueva Zelanda, Sudáfrica, Reino Unido y Estados Unidos.

El Acuerdo de Sydney se inició en 2001 y reconoce la equivalencia sustancial en la acreditación de las cualificaciones en la tecnología de la ingeniería, con una duración de tres años. [12]

También se encuentra el Acuerdo Latinoamericano Sobre Acreditación en Ingeniería (ALAI), que fue firmado en Argentina en el año 2010 por las agencias de los siguientes países: Argentina, Bolivia, Brasil, Chile, Colombia, México y Paraguay. [13]

\section{ORGANISMOS DE ACREDITACIÓN EN MÉXICO}

En México, en el año 2000 se creó el Consejo para la Acreditación de la Educación Superior (COPAES), quien hasta la fecha ha reconocido a 30 organizaciones de acreditación de diferentes disciplinas, de entre las cuales se pueden mencionar: el Consejo de Acreditación de la Enseñanza de la Ingeniería, A.C. (CACEI), Consejo de Acreditación de Programas Educativos en Física, A.C (CAPEF), Consejo de Acreditación de programas Educativos en Matemáticas A.C. (CAPEM), Consejo de Acreditación en Ciencias Administrativas Contables y Afines, A.C. (CACECA), entre otros. [14]

\section{A. $C A C E I$}

El Consejo de Acreditación de la Enseñanza de la Ingeniería, A.C. (CACEI), es la primera instancia acreditadora que se constituye en nuestro país. Fue formada en 1994 como asociación civil y cuya función es de gran trascendencia, pues coadyuva a la mejora de la calidad de la enseñanza de la ingeniería y proporciona información oportuna, pertinente y objetiva. El CACEI se rige actualmente por el documento "Marco de Referencia 2014 para la Acreditación de los Programas de Ingeniería", pero recién concluyó un nuevo Marco de Referencia (MR-2018), que está basado en estándares internacionales, como resultado de su ingreso al Washington Accord. El MR-2018 entrará en vigencia en enero de 2018. [15]

En 2015, CACEI y ANECA firmaron un convenio de colaboración específico para la acreditación del sello "EUR-ACE" en México, donde se tiene como objetivo ofrecer a los títulos (programas) de ingeniería de las universidades mexicanas, la posibilidad de obtener el sello EURACE a través de un proceso de acreditación. Donde se establece un proyecto piloto en el que participan tres universidades mexicanas: Universidad Autónoma de Yucatán, Universidad Autónoma de San Luis Potosí y Universidad Autónoma de Nuevo León, con dos Programas Educativos cada una. [16]

Desde 2014, CACEI encaminó sus esfuerzos a la incorporación al Washington Accord (WA), el acuerdo internacional de carácter educativo que reconoce a los mejores organismos acreditadores de ingeniería del mundo. Para lo cual se sometió a evaluación por parte de dos miembros signatarios del WA: ABET, de Estados Unidos, en abril de 2015 y CEAB, de Canadá, en octubre de ese mismo año, logrando el aval de ambos organismos y obteniendo la aceptación como miembro provisional en febrero de 2016. [17]

Debido a esto, CACEI se encontró en la necesidad de actualizar su Marco de Referencia para incorporar estándares internacionales, en junio de 2016 se dio a conocer el proyecto en la Conferencia Nacional de Facultades y Escuelas de Ingeniería (ANFEI). Con este documento, puede apreciarse la importancia que la Mejora Continua representa para los nuevos estándares de calidad, lo que eventualmente lleva a un aseguramiento de la misma. [18]

\section{B. CONACYT}

El Consejo Nacional de Ciencia y Tecnología fue creado en 1970, como organismo público "con el objetivo de consolidar un Sistema Nacional de Ciencia y Tecnología que responda a las demandas prioritarias del país, que dé solución a problemas y necesidades específicos, y que contribuya a elevar el nivel de vida y el bienestar de la población'"[10].

14 ${ }^{\text {th }}$ LACCEI International Multi-Conference for Engineering, Education, and Technology: "Engineering Innovations for Global Sustainability”, 20-22 July 2016, San José, Costa Rica. 
Como visión, el CONACYT tiene la contribución conjunta con otras dependencias y entidades del Gobierno Federal, así como del sector productivo para que México tenga una mayor participación en la generación adquisición y difusión del conocimiento a nivel internacional, y a que la sociedad aumente considerablemente su cultura científica y tecnológica, disfrutando de los beneficios derivados de ésta. [10]

Entre los múltiples programas del CONACYT, existe el Programa Nacional de Posgrados de Calidad (PNPC). En el documento "Marco de Referencia para la Evaluación y Seguimiento de Programas de Posgrado Presenciales", el CONACYT señala que la participación en el PNPC es un reconocimiento para las instituciones y centros de investigación que demuestran su capacidad de formación de posgrados, cumpliendo con los más altos estándares de pertinencia y calidad en el país. En el mismo, señala lo fundamental que es contar con un Sistema Interno de Aseguramiento de Calidad para cerciorarse de que la calidad es satisfactoria en cada etapa del proceso. [10]

\section{PROGRAMAS EDUCATIVOS MEXICANOS ACREDITADOS POR ORGANISMOS DE OTROS PAÍSES.}

En los últimos años se ha incrementado el número de universidades que optan por tener programas acreditados por organismos de otros países.

Actualmente existen seis programas educativos de tres universidades mexicanas que cuentan con el sello EUR-ACE obtenido a través de la Agencia Nacional de Acreditación de España (ANECA) [18].

Por otra parte, suman más de diez las universidades mexicanas que cuenta al menos con un programa acreditado por ABET, alcanzando un total de más de 70 programas educativos acreditados en México por dicha organización [19]. Es de mencionar que un número importante de programas educativos en México son susceptibles de acreditación por organismos de otros países, sin embargo, los costos de este tipo de acreditaciones es elevado y no todas las universidades cuentan con los recursos para este tipo de inversión.

\section{VII.LINEAMIENTOS GENERALES PARA LA ACREDITACIÓN DE UN SIAC A NIVEL INTERNACIONAL.}

El Sistema Interno de Gestión de Calidad representa un importante apoyo al momento de acreditar un programa educativo por una agencia diferente al país de origen. De hecho, es un requisito en los principales criterios que marcan organismos de otros países como ANECA y ENAEE. En la TABLA I se resumen los aspectos generales que contiene un Sistema Interno de Aseguramiento de Calidad, haciendo una comparativa con las normas, criterios y estándares que se revisan dependiendo de los organismos acreditadores.

\section{CONCLUSIONES}

La globalización, el crecimiento y la diversidad en la población estudiantil son las principales causas de la formación de diferentes tipos de organizaciones de educación superior, del aumento de la oferta educativa y de la inquietud de las escuelas por demostrar la calidad de su proceso educativo.
Las acreditaciones por parte de organismos externos, tanto internacional como nacionalmente, se vuelven cada vez más frecuentes con el objetivo de garantizar a la sociedad y alumnos que la calidad del sistema educativo es adecuada.

Las instituciones buscan el reconocimiento internacional con el objetivo de fomentar la movilidad de sus alumnos y sus maestros, al demostrar que cumplen con los mismos requisitos que otras universidades del mundo.

La Red Europea de Acreditación para la Enseñanza de la Ingeniería (ENAEE) es el grupo encargado de autorizar a organismos acreditadores la facultad de otorgar el sello EUR-ACE a los programas de ingeniería. El sello EUR-ACE es una garantía de calidad comparable con Europa de un programa educativo de ingeniería.

La Agencia Nacional de Evaluación de la Calidad y Acreditación de España (ANECA) es un organismo facultado por ENAEE para otorgar el sello EUR-ACE en España, México y América Latina. Dentro de los criterios que ENAEE propone para la obtención del sello EUR-ACE se encuentra contar con un Sistema Interno de Aseguramiento de Calidad.

En México, el principal organismo acreditador para los programas de ingeniería es el Consejo de Acreditación de la Enseñanza de la Ingeniería, A.C. (CACEI). A partir de 2015, CACEI ha comenzado a trabajar con ANECA para la acreditación de programas en distintas escuelas del país, por lo que contar con un Sistema Interno de Aseguramiento de la Calidad se volverá un requisito de gran ayuda para la acreditación por parte de este organismo.

Debido a la importancia de contar con una certificación en un contexto internacional, CACEI en junio de 2016 actualiza su Marco de Referencia utilizando como referencia los modelos de acreditación de ABET y CEAB y agrega un requisito específico para la mejora continua, tal como ABET y CEAB determinan en sus respectivos Estándares de Acreditación.

El Consejo Nacional de Ciencia y Tecnología (CONACYT), a pesar de ser un organismo acreditador para programas de posgrado, demuestra la importancia de contar con un Sistema Interno de Aseguramiento de Calidad para la consolidación de los objetivos del programa educativo y el cerciorarse de que los estándares mínimos de calidad se están cumpliendo y que se encuentran en continua mejora.

Actualmente catorce universidades mexicanas cuentan con al menos una acreditación por parte de ENAEE o ABET lo que representan el interés de las instituciones nacionales por demostrar la calidad en sus programas de ingeniería de forma global.

Finalmente, se puede concluir que la similitud entre las características de un Sistema Interno de Aseguramiento de Calidad y los requisitos por parte de los organismos acreditadores investigados en este trabajo, ya sea nacional o internacional, demuestran que un Sistema Interno de Aseguramiento de Calidad o un mecanismo similar, es la herramienta principal para el cumplimento de cada uno de sus estándares de calidad, lo que no sólo permitirá obtener la excelencia, y por ende la acreditación, sino también asegurará a mantenerla a lo largo del tiempo.

14 ${ }^{\text {th }}$ LACCEI International Multi-Conference for Engineering, Education, and Technology: "Engineering Innovations for Global Sustainability”, 20-22 July 2016, San José, Costa Rica. 
TABLA I

COMPARATIVA DE LOS LINEAMIENTOS GENERALES PARA LA ACREDITACIÓN DE UN SIAC

\begin{tabular}{|c|c|c|c|c|c|}
\hline Elementos de un Sistema Interno de Aseguramiento de Calidad & $\begin{array}{l}\text { ENAEE- } \\
\text { ANECA }\end{array}$ & ABET & CEAB & $\begin{array}{l}\text { CACEI } \\
(2014)\end{array}$ & $\begin{array}{l}\text { CACEI } \\
(2018)\end{array}$ \\
\hline \multicolumn{6}{|c|}{ Cultura de Calidad } \\
\hline Política & Criterio 1 & Estándar 1 & - & Categoría 3 & - \\
\hline Objetivos & Criterio 1 & Estándar 2 & - & Categoría 3 & - \\
\hline Procedimientos & Criterio 1 & - & - & Categoría 3 & - \\
\hline Transparencia & Criterio 1 & - & - & - & - \\
\hline Gobierno y gestión & Criterio 1 & - & Criterio 5 & Categoría 10 & - \\
\hline Planeación & Criterio 1 & - & - & Categoría 10 & - \\
\hline \multicolumn{6}{|c|}{ Recursos y Servicios } \\
\hline Infraestructura & Criterio 6 & Estándar 7 y 8 & Criterio 5 & Categoría 9 & Criterio 5 \\
\hline Tecnología & Criterio 6 & Estándar 7 & Criterio 5 & Categoría 9 & Criterio 5 \\
\hline Acceso y administración al conocimiento y a la información & Criterio 6 & Estándar 7 & Criterio 5 & Categoría 9 & Criterio 5 \\
\hline \multicolumn{6}{|c|}{ Información pública } \\
\hline Estadísticas de ingreso y egreso & Criterio 7 y 8 & - & - & - & - \\
\hline Estadísticas de Empleabilidad & Criterio 7 y 8 & - & - & - & - \\
\hline $\begin{array}{l}\text { Estadísticas sobre satisfacción de empleadores y otros grupos de } \\
\text { interés }\end{array}$ & Criterio 7 y 8 & - & - & - & - \\
\hline \multicolumn{6}{|c|}{ Diseño de la oferta formativa } \\
\hline Aprobación de oferta & Criterio 2 & Estándar 2 & Criterio 3 & Categoría 3 & Criterio 3 \\
\hline Control y supervisión de oferta & Criterio 2 y 9 & Estándar 2 & - & Categoría 3 & Criterio 3 \\
\hline Mecanismos de corrección en caso de incumplimiento & Criterio 2 y 9 & Estándar 4 y 5 & - & - & - \\
\hline $\begin{array}{l}\text { Resultados del proceso formativo (Aprendizaje, Inserción Laboral, } \\
\text { grupos de interés ) }\end{array}$ & Criterio 2,3 y 8 & Estándar 2, 3 y 4 & Criterio 2 & Categoría 4 y 7 & Criterio 3 \\
\hline \multicolumn{6}{|l|}{ 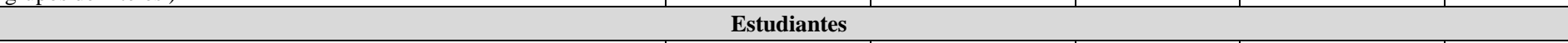 } \\
\hline Perfil de ingreso & Criterio 7 & - & - & Categoría 2 y 3 & Criterio 2 \\
\hline Admisión de estudiantes & Criterio 4 & Estándar 1 & Criterio 3 y 5 & Categoría 2 & Criterio 2 \\
\hline Servicios estudiantiles y complementarios & Criterio 4 y 6 & Estándar 6 y 7 & Criterio 3 y 5 & Categoría 3,5 y 6 & Criterio 6 \\
\hline Relación con el contexto & Criterio 5 & - & Criterio 1 y 5 & Categoría 2 & - \\
\hline Perfil de egreso & Criterio 4 & Estándar 1 & Criterio 1 y 3 & Categoría 3 & Criterio 3 \\
\hline \multicolumn{6}{|c|}{ Personal Académico y de apoyo a la docencia } \\
\hline Perfil del profesor & - & Estándar 6 & Criterio 5 & Categoría 1 & Criterio 1 \\
\hline Formación docente & Criterio 5 & Estándar 6 & Criterio 5 & Categoría 1 & Criterio 1 \\
\hline Modalidades de contratación del personal & Criterio 5 & - & - & Categoría 1 & Criterio 1 \\
\hline Servicios administrativos al servicio de la docencia & Criterio 5 & Estándar 7 & Criterio 5 & Categoría 10 & Criterio 6 \\
\hline Incentivos & - & - & Criterio 5 & Categoría 1 & Criterio 1 \\
\hline \multicolumn{6}{|c|}{ Investigación } \\
\hline Programas de creación de conocimiento & - & - & - & Categoría 8 & - \\
\hline Producción intelectual útil para el contexto & - & - & - & Categoría 8 & - \\
\hline $\begin{array}{l}\text { Mecanismos de divulgación de la investigación generada o aplicada, } \\
\text { diseñada para los grupos de interés }\end{array}$ & - & - & - & Categoría 8 & - \\
\hline \multicolumn{6}{|c|}{$\begin{array}{ll}\text { Proyección Social y Vinculación } \\
\end{array}$} \\
\hline Promoción de la colaboración con sectores de la sociedad. & - & - & Criterio 1 & Categoría 7 & - \\
\hline Rol de los estudiantes y el programa en los procesos de vinculación. & - & - & - & Categoría 7 & - \\
\hline \multicolumn{6}{|c|}{ Plan de Mejora } \\
\hline Implementación de acciones correctivas & Criterio 9 & Estándar 4 & Criterio 2 & - & Criterio 4 \\
\hline $\begin{array}{l}\text { Elaboración de estrategias, objetivos y metas encaminados a la } \\
\text { mejora continua }\end{array}$ & Criterio 9 & Estándar 4 & Criterio 2 & - & Criterio 4 \\
\hline $\begin{array}{l}\text { Procedimientos para la evaluación, el seguimiento y análisis de } \\
\text { resultado e impacto de acciones del plan de mejora }\end{array}$ & Criterio 9 & Estándar 4 & Criterio 2 & - & Criterio 4 \\
\hline $\begin{array}{l}\text { Participación de autoridades, profesores y estudiantes asociados al } \\
\text { programa. }\end{array}$ & Criterio 9 & Estándar 4 & Criterio 5 & - & - \\
\hline
\end{tabular}

Fuente: Comparativa realizada a partir de los marcos de referencia de cada una de las agencias.

14 ${ }^{\text {th }}$ LACCEI International Multi-Conference for Engineering, Education, and Technology: "Engineering Innovations for Global Sustainability", 20-22 July 2016, San José, Costa Rica. 


\section{REFERENCIAS BIBLIOGRÁFICAS}

[1] Centro Interuniversitario de Desarrollo (CINDA), Aseguramiento de la calidad en Iberoamérica. Educación Superior Informe 2012. Santiago, Chile. RIL editores. Universia, 2012. Consultado el 15 de marzo del 2016 en http://www.universia.net/nosotros/files/CINDA2012-Informe-de-Educación-Superior.pdf

[2] C. Bellei, Situación Educativa de América Latina y el Caribe: Hacia la educación de calidad para todos 2015. Santiago, Chile: UNESCO, 2015. Consultado el 13 de junio de 2016 en http://www.unesco.org/new/fileadmin/MULTIMEDIA/FIELD/Santia go/images/SITIED-espanol.pdf

[3] Secretaría de Educación Pública (SEP), Estadísticas Históricas por Escuelas, Alumnos y Maestros según el nivel educativo desde 1893 a 2013. Consultado el 31 de Octubre de 2016 en http://www.snie.sep.gob.mx/descargas/estadistica/SEN_estadistica_hi storica_nacional.pdf

[4] I. Dussel, Educación y nuevas tecnologías: Los desafíos pedagógicos ante el mundo digital. Buenos Aires, Argentina. Santillana. 2010. Consultado el 18 de Julio de 2016 en http://www.unsam.edu.ar/escuelas/humanidades/actividades/latapi/do cs/Dussel-Quevedo.pdf

[5] G. Coto, Determinación de un modelo homólogo para acreditación de carreras de Ingeniería de distintos países. Costa Rica. LACCEI 2007. Consultado el 17 de Junio de 2016 en http://www.laccei.org/LACCEI2007-Mexico/p137.doc

[6] O. Espinoza, Los sistemas de aseguramiento de la calidad en la educación superior en América Latina”, 2011. Consultado el 28 de marzo de 2016 en

http://www.revistaakademeia.cl/wp/wp-content/uploads/2010/08/Lossistemas-de-aseguramiento-de-la-calidad-en-la-educacion-superioren-America-Latina.pdf

[7] M. Morande, Sistema de aseguramiento de la calidad de la Educación Superior: aspectos críticos y desafios de mejoramiento. Pontificia Universidad Católica de Chile, 2011. Consultado el 2 de Junio de 2016 en http://politicaspublicas.uc.cl/wpcontent/uploads/2015/02/sistema-de-aseguramiento-de-la-calidad-dela-educacion-superior.pdf

[8] J. Aguilar, La mejora continua. Oaxaca, México. Conductitlán. 2010. Consultado el 31 de octubre de 2016 en http://www.conductitlan.net/psicologia_organizacional/la_mejora_co ntinua.pdf

[9] Criterios y directrices para el aseguramiento de Calidad en el Espacio Europeo de Educación Superior (ESG) Conferencia de Ministros. Armenia, 2015. Recuperado el 13 de junio de 2016 de http://www.aneca.es/content/download/13288/164541/file/ESG_caste $\underline{\text { llano_2015b.pdf }}$
[10] Documentos del PNPC No.1 Código de Buenas Prácticas" CONACYT, 2015. Recuperado el 3 de marzo de 2016 de http://www.conacyt.mx/index.php/becas-y-posgrados/programanacional-de-posgrados-de-calidad/convocatorias-avisos-yresultados/documentos/9004-codigo-de-buenas-practicas/file

[11] A. Roa, I. Pacheco, Educación Superior en Colombia: Doce Propuestas para la próxima década. BOSTON COLLEGE, Colombia, 2014. Pp. 270-273

[12] Washington Accord Homepage. International Engineering Alliance. Consultado el 31 de octubre de 2016 en http://www.ieagreements.org/Washington-Accord/signatories.cfm

[13] Ramos T. “Acreditación de programas de ingeniería en América Latina" en Regional Meeting 2012 IEEE, Ciudad de México, México, Abr. 12, 2012. Consultado el 7 de noviembre de 2016 en www.ewh.ieee.org/reg/9/eventos/rr2012/rrdocs/../3_acreditationadvis oryproject.pptx

[14] Consejo para la Acreditación de la Educación Superior. COPAES. "Organismos Acreditadores". Consultado el 7 de noviembre de 2016 en http://www.copaes.org/

[15] ¿Quiénes somos? Consejo de Acreditación de la Enseñanza de la Ingeniería. CACEI. 2016 Consultado el 31 de octubre de 2016 en http://cacei.org.mx/index.php/nosotros/quienes-somos

[16] M. Becerro El proceso de acreditación EUR-ACE de ANECA en América Latina. Nuevo León México, 2015. Consultado el 30 de Mayo de 2016 en

www.asibei.net/documentos/eventos/seminario porto 2015/aneca ac reditacion_eur-ace_america_latina.pdf

[17] El CACEI miembro provisional del Washington Accord. Pressreader. México, Mar. 3, 2016. Consultado el 31 de octubre de 2016 en

http://www.pressreader.com/mexico/milenio/20160303/28336047117 $\underline{8339}$

[18] Barrera M. "Nuevo marco de referencia del CACEI de acuerdo a los estándares internacionales" en XLIII Conferencia Nacional de la ANFEI, Nuevo León, México Jun. 19, 2016. Consultado el 31 de octubre de 2016 en http://www.anfei.mx/site/wp-content/uploads/2016/06/ConferenciaMEBB_CACEI.pdf

[19] ABET. (2016) ABET Accredited Program Search. Consultado en octubre 2016 en

http://main.abet.org/aps/Accreditedprogramsearch.aspx 